\title{
Cyberbullying escolar: incidencia del teléfono móvil e internet en adolescentes
}

\author{
Cyberbullying in schools: mobile phone and internet effect in adolescents
}

\author{
Domínguez-Alonso, José; Vázquez-Varela, Elia \& Nuñez-Lois, Sandra \\ Universidad de Vigo
}

\begin{abstract}
The study objective is focusing on the different cyberbullying forms (mobile phone and internet), and how these are influenced by the personal and scholar variables. The final sample was composed for a total of 749 students from the Secondary Education, between 12 and 15 years old $(\mathrm{M}=13.77$ years; DT $=1.12)$. To collect information, we use a survey "ad hoc" (data about social factors at school) and the Adolescent Victimization through Mobile Phone and Internet Scale (CYBVIC) (Buelga, Cava \& Musitu, 2012). In spite of obtaining a similar percentage with the first descriptive analysis, there is a soft prevalence of bulling via mobile phone (18.6\%) over bullying via internet (12\%) in teenagers. Likewise, among the different behaviours to assault someone, the denigration and the intimacy violation excel in both case, mobile phones and internet. Furthermore, data shows us that teenagers with more predispositions to suffer cyberbullying via mobile phone and internet are girls between thirteen and fifteen years old, who failed some subject and with an unstructured family.
\end{abstract}

Reception Date 2017 June 29

Approval Date 2017 November 16

Publication Date: 2017 November 17

\section{Keywords:}

Cyberbullying; adolescence; mobile phone; internet

\begin{abstract}
Resumen
El objetivo del estudio está enfocado a conocer la prevalencia de las formas de cyberbullying (teléfono móvil e internet) y cómo estas se ven influenciadas por las variables personales y escolares. La muestra final estuvo formada por un total de 749 alumnos de Educación Secundaria Obligatoria, con edades comprendidas entre los 12 y los 15 años $(M=13.77$ años; DT = 1.12). Para la recogida de información se utiliza un cuestionario "ad hoc" (datos socioescolares) y la escala de Victimización entre Adolescentes a través del Teléfono Móvil y de Internet (CYBVIC) (Buelga, Cava \& Musitu, 2012). De un primer análisis descriptivo, se obtienen porcentajes similares pero con ligera prevalencia en los actos de acoso a través del teléfono móvil (18.6\%) sobre internet (12\%) de los adolescentes. Asimismo, entre las formas de comportamiento que implican agresiones, tanto a través del teléfono móvil e internet, despuntan la denigración y la violación de la intimidad. Además, los datos indican que los adolescentes con mayor predisposición a sufrir cyberbullying a través del teléfono móvil e internet serían chicas entre trece y quince años, con alguna materia suspensa y que viven en familias desestructuradas.
\end{abstract}

\section{Palabras clave:}

Cyberbullying; adolescencia; teléfono móvil; Internet

Fecha de recepción 2017 Junio 29

Fecha de aprobación 2017 Noviembre 16

Fecha de publicación 2017 Noviembre 17
Los incesantes cambios sociales de los últimos años, la creciente conflictividad social, el impacto de sucesos terribles (suicidios), así como su repercusión sobre actitudes personales y relacionales, han puesto de relieve la necesidad de contemplar el fenómeno del cyberbullying en la agenda educativa. Los peligros derivados del mismo, son padecidos por todos los ciudadanos, y se acentúan en mayor medida en el periodo 
adolescente (Amado, Matos, Pessoa y Jäger, 2009; Del-Rey, Casas \& Ortega, 2012; Garaigordobil, 2011; Livingstone, Haddon, Görzig \& Ólafsson, 2011). Además, es constatable, en las últimas décadas, una nueva brecha digital entre quienes tienen una concepción y uso correcto de las tecnologías de la información y comunicación y quienes no lo tienen (Duart, 2010), la cual tiende a crecer entre determinados colectivos sociales, sobre todo en adolescentes.

Aun poniendo de relevancia que las tecnologías de la información y comunicación (sobre todo el teléfono móvil e internet) están destinadas a mejorar las relaciones humanas, también es cierto que no siempre se hace un uso adecuado de ellas. Se da la paradoja de que, aunque se le sigue atribuyendo un notable potencial de ascendencia social, resulta contradictorio que sean reconocidas como un problema emergente de salud pública (David-Ferdon \& Feldman, 2007; Juvonen \& Gross, 2008). No obstante, conseguir delimitar por completo su uso es una tarea tan imposible como inútil, especialmente en los tiempos actuales en los que se asiste a una total dependencia e incremento constante en su empleo.

Por ello, es lógico que el punto de partida sea el reconocimiento del cyberbullying, entendido como una conducta agresiva e intencional que se repite de forma frecuente en el tiempo mediante el uso, por un individuo o grupo, de dispositivos electrónicos sobre una víctima que no puede defenderse (Belsey, 2005; Smith, Mahdavi, Carvalho, Fisher, Russell \& Tippett, 2008). En este sentido, aunque el componente con mayor peso en el cyberbullying sea el bullying (Campbell, 2005), presenta matices que lo hacen más pernicioso, entre los que destaca la garantía del anonimato y la masificación de la agresión, acarreando un incremento en la indefensión de la víctima (Hernández \& Solano, 2007). No obstante, independientemente de que el constructo de cyberbullying es complejo y difícil de operacionalizar (Garaigordobil, 2013), los comportamientos que lo conforman se pueden agrupan en: verbales y escritos (e-mails, chats, blogs, websites), visuales (compartir, publicar y enviar fotos o videos), suplantación (robar o revelar información personal), y exclusión (aislar deliberadamente a alguien) (Nocentini et al., 2010).

Así pues, el cyberbullying, es la forma que adquiere el fenómeno de acoso entre iguales modernizado, sesgado por el incesante impacto que las nuevas tecnologías de la información y comunicación ejercen en comportamientos y relaciones interpersonales de adolescentes (Calmaestra, 2011; MoraMerchán, 2008). Probablemente, muchas situaciones de bullying se sirvan de estas nuevas tecnologías (TIC) para mudar a un nuevo fenómeno llamado cyberbullying (Smith, Mahdavi, Carvalho \& Tippett, 2006). Esta atribución, lleva a entenderlo como la consecución de comportamientos agresivos e insultantes contra una persona a través de tecnologías interactivas, básicamente telefonía móvil e internet (Avilés, 2009; Buelga, Cava \& Musitu, 2010; Ortega, Calmaestra \& MoraMerchán, 2008).

Incidiendo en esta mayor preocupación por el cyberbullying en los centros educativos, hay que indicar aspectos particulares de este tipo de violencia relacional que le lleva a ser más peligroso, como son: la sensación de estar siempre atrapado, la invisibilidad de los agresores, indefensión de la víctima, difusión masiva de las cyberagresiones, y sobre todo, por la amplitud del escenario (Buelga, Cava \& Musitu, 2012; Cerezo-Ramírez, 2012; McKenna, 2007). De igual manera, otro de los problemas que presenta el cyberbullying es su ocultación, destacando como principales motivos, el temor a ver limitado su acceso a las TIC, la vergüenza de las conductas realizadas ante sus padres, y sobre todo, la necesidad de arreglar ellos mismos sus propios problemas (Hoff \& Mitchell, 2009; Juvonen \& Gross, 2008; Kowalski \& Limber, 2007; Li, 2010). Por otro lado, Willard (2006, 2007) identifica como conductas agresivas a través de las tecnologías de la información y comunicación: hostigamiento, denigración, suplantación de identidad, violación de 
intimidad, exclusión social y cyberpersecución. En consecuencia, difícil de detectar y complicado de demostrar, así es el cyberbullying, que aunque a veces pueda ser imperceptible, es capaz de amargar la vida a muchos adolescentes.

En este escenario, se asiste a una creciente preocupación social y educativa por el potencial uso inapropiado y excesivo de internet entre los adolescentes (Blaszczynsky, 2006; Viñas, 2009). Los diferentes estudios evidencian la relevancia del fenómeno y su rápido crecimiento con porcentajes que oscilan entre el 10\% y el 30\% (Buelga, Cava \& Musitu, 2010; Cerezo, 2009; Garaigordobil \& Oñederra, 2008; Estévez, Villardón, Calvete, Padilla \& Orue, 2010; SánchezLacasa \& Cerezo, 2010; Perren, Dooley, Shaw \& Cross, 2010). Así pues, las investigaciones realizadas hasta el momento, solamente han permitido obtener una caracterización del problema, distinguiéndolo de otros tipos de violencia escolar.

Aun teniendo en cuenta el espíritu pacífico de la mayor parte de la población adolescente, es necesario promover programas de sensibilización, actuación, formación y seguimiento que ayuden a instaurar formas de convivencia positivas (Gairín, Armengol \& Silva, 2013). En este estudio se ha optado por considerar el cyberbullying según la vía por la que se realiza, diferenciando entre el desarrollado a través del teléfono móvil (hostigamiento, denigración, violación de intimidad y exclusión social) y el llevado a cabo a través de internet (hostigamiento, denigración, violación de intimidad, exclusión social y suplantación de identidad). Por lo tanto, el objetivo del estudio está enfocado a conocer la prevalencia de las formas de cyberbullying (teléfono móvil e internet) y cómo estas se ven influenciadas por las variables personales (edad, sexo y estructura familiar) y escolares (ubicación del centro educativo, curso escolar y rendimiento académico).

\section{Método}

\section{Participantes}

El tamaño de la población es de aproximadamente 88529 estudiantes (Xunta de Galicia, 2014). Utilizando el STATS, tendríamos que un tamaño de muestra adecuado para esta población (95\% de confianza, $5 \%$ de error y $p=0.5$ o $50 \%$ ) es de 383. La muestra final estuvo formada por un total de 749 alumnos de ESO, pertenecientes a 10 centros educativos (53\% mujeres; $47 \%$ hombres), con edades comprendidas entre los 12 y los 15 años ( $M=13.77$ años; $D T=1.12)$. La mayoría de los participantes vive en familias estructuradas (73.8\%), siempre ha aprobado (63.2\%), y estudia en centros urbanos (55.3\%). Además, presenta una distribución equilibrada en los cuatro cursos académicos: $1^{\circ}$ ESO (25.5\%), $2^{\circ}$ ESO (25.8\%), $3^{\circ}$ ESO (24.2\%), y $4^{\circ} \mathrm{ESO}$ (24.6\%).

\section{Instrumento}

Para la recogida de información se utiliza un cuestionario "ad hoc" (datos socioescolares) y la escala de Victimización entre Adolescentes a través del Teléfono Móvil y de Internet (CYBVIC) (Buelga, Cava \& Musitu, 2012). El primer instrumento recoge datos de identificación (datos personales y de los centros educativos) y el segundo consta de 18 ítems que miden en un rango de respuesta (nunca, pocas veces, muchas veces y siempre), la victimización experimentada a través del teléfono móvil (8 ítems) y de internet (10 ítems). La consistencia interna (alfa de Cronbach), en su versión original fue de .92 (.76 en el factor “teléfono móvil”, y .84 para internet).

\section{Procedimiento}

El estudio realizado fue aprobado por el Comité Ético de Investigación de nuestra institución. Seleccionada la muestra y los cuestionarios a utilizar, se contacta con el equipo directivo y los orientadores de los centros, con el objeto de explicarles la finalidad y alcance de la investigación y proponerles su participación voluntaria. Obtenido el consentimiento de la dirección de 
los centros, se mantiene una reunión informativa para dar a conocer el estudio, la garantía de su anonimato y que éstos lo trasladen a toda la comunidad educativa. Los tutores participan de forma voluntaria y no remunerada. Paralelamente, se informa a los padres sobre la investigación y se obtiene el consentimiento de la participación de sus hijos en la investigación.

\section{Análisis de datos}

La información recogida se trato con técnicas de análisis cuantitativas, descriptivas, inferenciales y confirmatorias, a través del programa SPSS 22. En primer lugar se analizaron las características técnicas del instrumento de medida (CYBVIC): análisis factorial exploratorio (AFE), confirmatorio (AFC), correlacional y análisis de fiabilidad (Alfa de Cronbach). En segundo lugar, se llevó a cabo el análisis descriptivo e inferencial: análisis de frecuencias y porcentajes, puntuaciones medias $\mathrm{y}$ desviación típica, análisis de varianza (ANOVA), y comparaciones múltiples a posteriori (Prueba Scheffé).

\section{Resultados}

\section{Validez y fiabilidad del cuestionario CYBVIC}

En un primer momento, se contrasta la validez (factorial y de criterio) y fiabilidad (consistencia interna) de la escala de Victimización entre adolescentes a través del teléfono móvil y de internet (CYBVIC). El análisis factorial exploratorio (AFE) proporcionó la dimensionalidad de la escala con un ajuste adecuado $\left(K M O=.892 ; \chi^{2}\right.$ : 4377.396; $G l=153 ; p<.001)$, arrojando dos factores que en su conjunto explican el $50.35 \%$ de la varianza total, dando cuenta de la estructura factorial original del instrumento. Seguidamente, a través del programa AMOS, se confirma la bidimensionalidad de la escala, comparando el grado de ajuste de nuestro modelo de dos factores (M2F) con el obtenido por Buelga, Cava y Musitu (2012). Los resultados (tabla 1) muestran un ajuste inferior al original, pero con unos índices de bondad de ajuste adecuados $\left(\chi^{2} / g l<3 ; C F I \geq .90 ; R M S E A \leq\right.$ $.05)$.

Tabla 1. Índices de bondad de ajuste de los dos modelos (original y puesto a prueba) de la escala CYBVIC, con el total de la muestra $(N=749)$

\begin{tabular}{ccccccc}
\hline Modelo & $\chi^{2}$ & $\boldsymbol{G l}$ & $\boldsymbol{P}$ & $\chi^{2} / \boldsymbol{g l}$ & $\boldsymbol{C F I}$ & RMSEA (IC 90\%) \\
\hline M2FO & 359.11 & 131 & .000 & 2.74 & .91 & $.03(.02-.03)$ \\
M2FC & 1112.65 & 398 & .000 & 2.79 & .90 & $.05(.03-.06)$ \\
\hline
\end{tabular}

$\chi^{2}$ : Chi-cuadrado; gl: grados de libertad; p: nivel de probabilidad; CFI: índice de ajuste comparativo; RMSEA: error cuadrado medio de aproximación; IC: intervalo de confianza; M2FO: modelo de dos factores original; M2FC: modelo de dos factores a confirmar.

Una vez identificada la bidimensionalidad de la escala, se analizó la correlación (validez de criterio) entre la puntuación total en el CYBVIC y sus dos criterios externos sobre los que existe evidencia previa de su asociación con el cyberbullying. Así, la correlación entre las dos dimensiones (teléfono móvil e Internet) fue de .71 (correlación de Pearson), lo cual confirmó la existencia de una alta relación entre los factores. Por último, se analizó la fiabilidad de la escala en términos de consistencia interna (alpha de Cronbach). El alpha para el conjunto de la escala es de 87 (buena), con valores de .75 para el teléfono móvil y .79 para Internet.

\section{Prevalencia del teléfono móvil o internet en el cyberbullying adolescente}

En primer lugar, se lleva a cabo un análisis descriptivo a través de los porcentajes de la 
totalidad de los ítems que conforman el cyberbullying. De este análisis se deduce que un $18.6 \%$ de los adolescentes consuman el acoso a través del teléfono móvil, frente a un $12 \%$ que lo hace a vía internet. Asimismo, el $84.7 \%$ considera que nunca ha practicado cyberbullying, un $11.6 \%$ lo hace a veces, y el $3.7 \%$ lo realiza habitualmente. Así pues, el análisis de resultados indica que un $15.3 \%$ de los adolescentes considera que se producen este tipo de agresiones (figura 1).

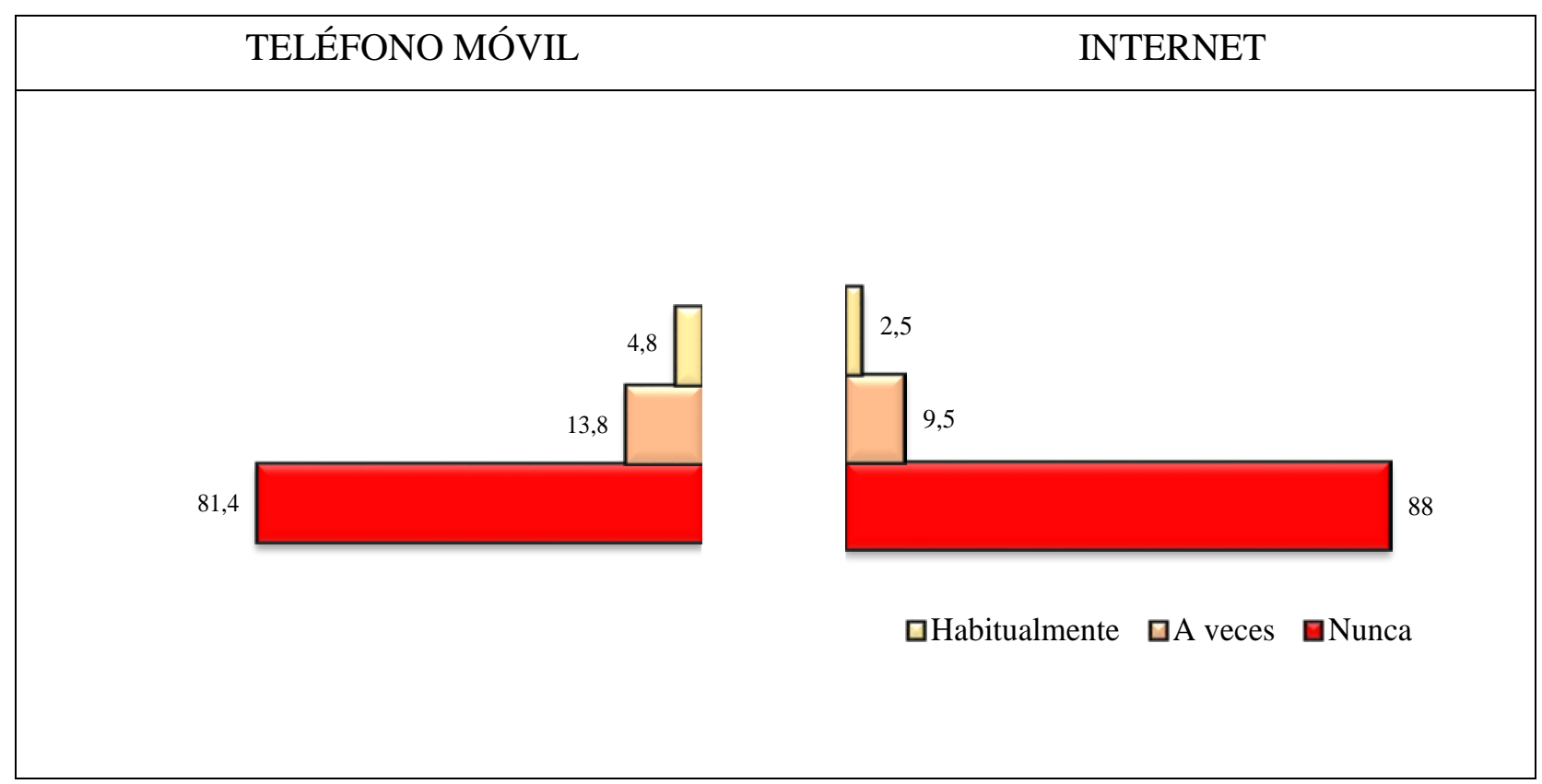

Figura 1. Porcentajes del cyberbullying a través del teléfono móvil e internet

Si se atiende a las formas en las que se lleva a cabo el cyberbullying a través de las redes sociales (teléfono móvil e internet), destacan la denigración (Han contado mentiras o rumores falsos sobre mí. Tm: 39\%; I: 25\%), y la violación de la intimidad (Han compartido mis secretos con otros. Tm: 31\%; I: 19\%). Por el contrario, las menos habituales son el hostigamiento (Me han obligado a hacer cosas que no quería con amenazas. Tm: 4\%; I: 3\%), y la suplantación de identidad (Han pasado y manipulado fotos o videos de mí o de mi familia sin mi permiso. Tm: 6\%; I: 6\%).

Análisis descriptivo e inferencial de las variables personales

A continuación se procede a medir el efecto del cyberbullying a través del teléfono móvil e internet en relación a la edad, sexo, y estructura familiar, estudiando las puntuaciones medias, desviaciones típicas y nivel de significatividad (tabla 2). 
Tabla 2. Medias, desviaciones típicas y análisis de varianza atendiendo a la edad, sexo, y tipo de familia

\begin{tabular}{|c|c|c|c|c|c|c|c|c|}
\hline \multirow{2}{*}{$\begin{array}{l}\text { VARIABLES } \\
\text { PERSONALES }\end{array}$} & \multicolumn{4}{|c|}{ TELÉFONO MÓVIL } & \multicolumn{4}{|c|}{ INTERNET } \\
\hline & Media & $D T$ & $\boldsymbol{F}$ & Sig. & Media & $D T$ & $\boldsymbol{F}$ & Sig. \\
\hline \multicolumn{9}{|c|}{ EDAD } \\
\hline 12 años & 9.32 & 2.39 & \multirow{4}{*}{4.26} & \multirow{4}{*}{.005} & 10.68 & 1.94 & \multirow{4}{*}{6.26} & \multirow{4}{*}{.000} \\
\hline 13 años & 10.16 & 2.78 & & & 11.67 & 3.23 & & \\
\hline 14 años & 9.89 & 2.34 & & & 11.59 & 2.54 & & \\
\hline 15 años & 10.29 & 2.91 & & & 11.93 & 2.99 & & \\
\hline \multicolumn{9}{|c|}{ SEXO } \\
\hline Mujer & 10.16 & 2.69 & \multirow{2}{*}{3.48} & \multirow{2}{*}{.049} & 11.66 & 2.54 & \multirow{2}{*}{1.05} & \multirow{2}{*}{.306} \\
\hline Hombre & 9.80 & 2.71 & & & 11.45 & 3.08 & & \\
\hline \multicolumn{9}{|c|}{ ESTRUCTURA FAMILIAR } \\
\hline Estructurada & 9.85 & 2.61 & \multirow{2}{*}{5.33} & \multirow{2}{*}{.021} & 11.47 & 2.79 & \multirow{2}{*}{2.42} & \multirow{2}{*}{.120} \\
\hline Desestructurada & 10.37 & 2.94 & & & 11.83 & 2.83 & & \\
\hline
\end{tabular}

Con los datos obtenidos en las medias, la mayor probabilidad de cyberbullying a través del teléfono móvil e internet por parte del alumnado se produciría en jóvenes de quince años $(M t m=10.29 ; M i=11.93)$, de sexo femenino $(M t m=10.16 ; M i=11.66), \mathrm{y}$ conviven en familias desestructuradas $(\mathrm{Mtm}=$ 10.37; $M i=11.83)$. Por el contrario, los adolescentes con menor probabilidad de cyberbullyin a través del teléfono móvil e internet serían hombres $(\mathrm{Mtm}=9.80 ; \mathrm{Mi}=$ 11.45), de doce años $(M t m=9.32 ; M i=$ 10.68), y viven en familias estructuradas $(M t m=9.85 ; M i=11.47)$. Asimismo, el análisis de varianza muestra que las variables edad $(F=4.26 ; p=.005)$, sexo $(F=3.48 ; p=$ .049), y estructura familiar $(F=5.33 ; p=$ .021), son fuentes significativas de variación con respeto a la variable cyberbullyin a través del teléfono móvil; mientras que solamente la variable edad $(F=6.26 ; p \leq .001)$ es significativa con respecto a la variable cyberbullying a través de internet.

Confirmados los resultados del ANOVA, se lleva a cabo un análisis a posteriori con las variables significativas a través de la prueba
Scheffé. Así, muestran diferencias significativas (cyberbullying a través del teléfono móvil) en la variable edad, entre los que tienen 13 y 15 años con los de 12 años; sexo, entre el femenino con el masculino; $\mathrm{y}$ estructura familiar, entre las familias desestructuradas con las estructuradas. Además, evidencias diferencias significativas (cyberbullying a través de internet) en la edad, entre los que tienen 13, 14 y 15 años con los de 12 años. Estos resultados sugieren una mayor probabilidad de cyberbullying a través del teléfono móvil en chicas adolescentes de trece y quince años que viven en familias desestructuradas. Igualmente, el cyberbullying a través de internet es superior a mayor edad.

Análisis descriptivo e inferencial de las variables escolares

Seguidamente, se procede a medir el efecto del cyberbullying a través del teléfono móvil e internet en relación al centro educativo, curso escolar, y rendimiento académico, estudiando las puntuaciones medias, desviaciones típicas y nivel de significatividad (tabla 3). 
Tabla 3. Medias, desviaciones típicas y análisis de varianza atendiendo a la ubicación del centro, curso, y rendimiento académico

\begin{tabular}{|c|c|c|c|c|c|c|c|c|}
\hline \multirow{2}{*}{$\begin{array}{l}\text { VARIABLES } \\
\text { ESCOLARES }\end{array}$} & \multicolumn{4}{|c|}{ TELÉFONO MÓVIL } & \multicolumn{4}{|c|}{ INTERNET } \\
\hline & Media & $D T$ & $\boldsymbol{F}$ & Sig. & Media & $D T$ & $\boldsymbol{F}$ & Sig. \\
\hline \multicolumn{9}{|c|}{ UBICACIÓN DEL CENTRO } \\
\hline Villa & 10.41 & 3.03 & \multirow{2}{*}{14.37} & \multirow{2}{*}{.000} & 11.91 & 2.76 & \multirow{2}{*}{8.71} & \multirow{2}{*}{.003} \\
\hline Ciudad & 9.65 & 2.35 & & & 11.29 & 2.83 & & \\
\hline \multicolumn{9}{|c|}{ CURSO ESCOLAR } \\
\hline $1^{\circ}$ ESO & 9.69 & 2.56 & \multirow{4}{*}{2.43} & \multirow{4}{*}{.047} & 11.18 & 2.41 & \multirow{4}{*}{2.67} & \multirow{4}{*}{.047} \\
\hline $2^{\circ}$ ESO & 10.17 & 2.82 & & & 11.65 & 3.05 & & \\
\hline $3^{\circ}$ ESO & 9.78 & 2.37 & & & 11.46 & 2.55 & & \\
\hline $4^{\circ}$ ESO & 10.32 & 2.99 & & & 11.97 & 3.12 & & \\
\hline \multicolumn{9}{|c|}{ RENDIMIENTO ACADÉMICO } \\
\hline Aprueba & 9.81 & 2.63 & & & 11.35 & 2.56 & & \\
\hline Algún suspenso & 10.43 & 3.11 & 3.22 & .041 & 12.06 & 3.88 & 4.06 & .018 \\
\hline Repite & 10.21 & 2.52 & & & 11.84 & 2.45 & & \\
\hline
\end{tabular}

Con los datos obtenidos en las medias, la mayor probabilidad de cyberbullying a través del teléfono móvil e internet se produciría en villas $(M t m=10.41 ; M i=11.91)$, cursando cuarto de ESO $(M t m=10.32 ; M i=11.97)$, y en su vida académica han suspendido alguna materia $(M t m=10.43 ; M i=12.06)$. Por el contrario, los adolescentes con menor probabilidad de cyberbullyin a través del teléfono móvil e internet vive en ciudades $(M t m=9.65 ; M i=11.29)$, cursan primero de ESO $(M t m=9.69 ; M i=11.18)$, y siempre han aprobado $(\mathrm{Mtm}=9.81 ; \mathrm{Mi}=11.35)$. Asimismo, el análisis de varianza muestra que las variables ubicación del centro $(F=14.37$; $p \leq .001 ; F=8.71 ; p=.003)$, curso escolar $(F$ $=2.43 ; p=.047 ; F=2.67 ; p=.047), \mathrm{y}$ rendimiento académico $(F=3.22 ; p=.041 ; F$ $=4.06 ; p=.018$ ), son fuentes significativas de variación con respeto a la variable cyberbullyin a través del teléfono móvil e internet.

Confirmados los resultados del ANOVA, se lleva a cabo un análisis a posteriori con las variables significativas a través de la prueba Scheffé. Así, muestran diferencias significativas (cyberbullying a través del teléfono móvil e internet) en la variable ubicación centro, entre los que estudian en villas con los que lo hacen en ciudades; curso escolar, entre los que cursan cuarto de ESO con los de primero de ESO; y rendimiento académico, entre los que han suspendido alguna materia con los que siempre han aprobado todo. Estos resultados sugieren una mayor probabilidad de cyberbullying a través del teléfono móvil e internet en las villas, cursos elevados ( $4^{\circ}$ de ESO), y con alguna materia suspensa.

\section{Discusión}

Partiendo de un concepto amplio y dinámico, se entiende el cyberbullying como una nueva forma de bullying que implica el uso del teléfono móvil, internet u otras tecnologías de la información y comunicación para acosar, amenazar o intimidar deliberadamente a alguien (Baruch, 2005; Calmaestra, 2011). Así pues, considerando que el cyberbullying se puede enfocar por la vía que se produce el acoso (Dehue, Bolman, \& Vollink, 2008; Perren, Dooley, Shaw \& Cross, 2010), o por la conducta que se realice (Buelga, Cava \& Musitu, 2010), en este estudio se ha optado por la primera. Además, su acelerado desarrollo y expansión, sobre todo en poblaciones adolescentes, ha generado la urgente necesidad de estudio. En consecuencia, el propósito de esta investigación fue conocer el fenómeno del 
cyberbullying, identificando su frecuencia de uso y medio utilizado (teléfono móvil e Internet) para una mejor comprensión del problema, cuya relevancia en los centros educativos es indiscutible.

Esta investigación confirmó evidencias empíricas que apoyan la validez y fiabilidad de la Escala de Victimización entre Adolescentes a través del Teléfono Móvil e Internet (CYBVIC). La estructura teórica definida por dos factores relacionados fue confirmada por el análisis factorial exploratorio y ratificada por el análisis confirmatorio, relevando en ambos casos que el modelo se ajustaba adecuadamente a los datos (similar al original). También se obtuvieron evidencias de validez de constructo al ser todas las correlaciones estadísticamente significativas $(p \leq .001)$ y de signo positivo. Finalmente, el análisis de fiabilidad reveló un valor de .87, lo que indica una consistencia interna buena y que el instrumento puede aplicarse en otros contextos. En consecuencia la escala CYBVIC presenta propiedades psicométricas adecuadas que hacen recomendable su aplicación en la población adolescente (Buelga, Cava \& Musitu, 2012).

De un primer análisis descriptivo general, se obtienen porcentajes similares pero con ligera prevalencia en los actos de acoso a través del teléfono móvil (18.6\%) sobre internet (12\%) de los adolescentes, quizás justificado por la fusión de ambos en los nuevos teléfonos inteligentes (smartphones) (Álvarez-García, Dobarro \& Núñez, 2015). Estos resultados corroboran los estudios de Smith Mahdavi, Carvalho y Tippett (2006) y Guarini et al. (2009) que sostienen mayor frecuencia en agresiones por medio del teléfono móvil. No obstante refutan los realizados por Li (2007) y Wright, Burnham, Inman y Ogorchock (2009) que señalan más frecuentes las realizadas vía internet. Asimismo, entre las formas de comportamiento que implican agresiones, tanto a través del teléfono móvil e internet, despuntan la denigración y la violación de la intimidad. Por el contrario, las menos habituales son el hostigamiento, y la suplantación de identidad.

En segundo lugar, los datos indican que los adolescentes con mayor predisposición a sufrir cyberbullying a través del teléfono móvil e internet, serían chicas entre trece y quince años que viven en familias desestructuradas. En esta misma línea, los trabajos de Burgess-Proctor, Patchin e Hinduja (2009), Wade y Beran (2011), y Garaigordobil y Aliri (2013), sostienen que las chicas son más victimizadas que los chicos. No obstante, estudios como los de Calvete, Orue, Estévez, Villardón y Padilla (2010), Yilmaz (2011), y Pelfrey y Weber (2013), mantiene una mayor prevalencia en chicos. Por otro lado, el ciberbullying a través de internet es superior a mayor edad, no resultando significativa en el teléfono móvil. Ello coincide con la investigación de Ortega, Calmaestra y Mora-Merchán (2008) que considera la variable edad como no significativa. Por el contrario, los adolescentes con menor probabilidad de cyberbullying a través del teléfono móvil e internet serían hombres, de doce años, y viven en familias estructuradas.

Finalmente, el ciberbullying a través del teléfono móvil e internet en relación a las variables escolares, la mayor probabilidad se produciría en centros de villas (más de 10.000 habitantes), cursos elevados ( $4^{\circ}$ de ESO), y en alumnado con alguna materia suspensa. Aquí, la investigación presenta resultados contradictorios con otros estudios que afirman la tendencia a ser más victimizados los primeros cursos de ESO (Buelga, Cava \& Musitu, 2010; Buelga, Musitu, Murgui \& Pons, 2008; Díaz-Aguadó, 2005). Por otro lado, los adolescentes con menor probabilidad de cyberbullying a través del teléfono móvil e internet viven en ciudades (más de 100.000 habitantes), cursan primero de ESO, y siempre han aprobado.

Sería importante, trabajar desde la prevención e intervención inmediata, reforzando la vigilancia para minimizar su impacto (Ruiz, Riuró \& Tesouro, 2015). En último lugar, indicar que los resultados del trabajo deben asumirse con cautela, por los 
efectos de deseabilidad social, sesgos y transversalidad de los autoinformes utilizados. Pese a estas restricciones, el estudio invita a ahondar en esta problemática del cyberbullying, considerada como un nuevo tipo de violencia en la socialización de los adolescentes.

\section{Referencias}

Alvarez-García, D., Dobarro, A. \& Núñez, J. C. (2015). Validez y fiabilidad del Cuestionario de cibervictimización en estudiantes de Secundaria. Aula Abierta, 43, 32-38. doi: http://dx.doi.org/10.1016/j.aula.2014.11.001

Amado, J., Matos, A., Pessoa, T y Jäger, T. (2009). Cyberbullying: um desafio à investigação e à formação. Revista Interacções, 5(13), 301-326.

Avilés, J. M. (2009). Ciberbullying. Diferencias entre el alumnado de Secundaria. Boletín de Psicología, 96, 7996.

Baruch, Y. (2005). Bullying on the Net: Adverse behavior on email and its impact. Information and Management, 42, 361-371. doi: https://doi.org/10.1016/j.im.2004.02.001

Belsey, B. (2005). Cyberbullying: An emerging Threat to the "always on" generation. Recuperado de http://www.cyberbullying.ca

Blaszczynski, A. (2006). Internet use: In search of an addiction. International Journal of Mental Health and Addiction, 4, 7-9. doi: https://doi.org/10.1007/s11469-006-9002-3

Buelga, S., Cava, M. J., \& Musitu, G. (2010). Cyberbullying: victimización entre adolescentes a través del teléfono móvil y de Internet. Psicothema, 22(4), 784-789. doi: https://doi.org/10.1590/S102049892012000700006

Buelga, S., Cava, M. J. \& Musitu, G. (2012). Validación de la Escala de victimización entre adolescentes a través del teléfono móvil y de Internet. Revista Panamericana de Salud Publica, 32(1), 36-42.

Buelga, S., Musitu, G., Murgui, S. \& Pons, J. (2008). Reputation and aggressive behavior in adolescence. The Spanish Journal of Psychology, 11(1), 192-200. doi: https://doi.org/10.1017/S1138741600004236
Burgess-Proctor, A., Patchin, J. W. \& Hinduja, S. (2009). Cyberbullying and online harassment: Reconceptualizing the victimization of adolescent girls. En V. García \& J. Clifford (Eds.), Female crime victims: Reality reconsidered (pp. 153-175). Upper Saddle River, NJ: Prentice Hall.

Calmaestra, J. (2011). Cyberbullying: prevalencia y características de un nuevo tipo de bullying indirecto. Córdoba: Servicio de Publicaciones Universidad de Córdoba. Tesis doctoral.

Calvete, E., Orue, I., Estévez, A., Villardón, L. \& Padilla, P. (2010). Cyberbullying in adolescents: Modalities and aggressors' profile. Computers in Human Behavior, 26(5), 1128-1135. doi: https://doi.org/10.1016/j.chb.2010.03.017

Campbell, M. A. (2005). Cyberbullying: An old problem in a new guise? Australian Journal of Guidance and Counselling, 15(1), 68-76. doi: https://doi.org/10.1375/ajgc.15.1.68

Cerezo, F. (2009). Bullying: Análisis de la situación en las aulas españolas. International Journal of Psychology and Psychological Therapy, 9(3), 383-395.

Cerezo-Ramírez, F. (2012). Psique. Bullying a través de las TIC. Boletín Científico Sapiens Research, 2(2), 24-29.

David-Ferdon, C. \& Feldman, M. (2007). Electronic media, violence and adolescents: An emerging public health problem. Journal of Adolescent Health, 41(6, Supplement 1), S1-S5.

doi: https://doi.org/10.1016/j.jadohealth.2007.08. $\underline{020}$

Dehue, F., Bolman, C. \& Vollink, T. (2008). Cyberbullying: Youngsters' experiences and parental perception. CyberPsychology \& Behavior, 11(2), 217-223. doi: https://doi.org/10.1089/cpb.2007.0008

Del Rey, R., Casas, J. \& Ortega, R. (2012). El programa ConRed, una práctica basada en la evidencia. Comunicar, 39, 129-138. doi: http://dx.doi.org/10.3916/C39-2012-03-03

Díaz-Aguadó, M. J. (2005). La violencia entre iguales en la adolescencia y su prevención desde la escuela. Psicothema, 4(17), 549558.

Estévez, A., Villardón, L. Calvete, E., Padilla, 
P. \& Orue, I. (2010). Adolescentes víctimas de cyberbullying: prevalencia y características. Psicología Conductual, 18(1), 73-89.

Gairín, J., Armengol, C. \& Silva, B.P. (2013). El "bullying” escolar. Consideraciones organizativas y estrategias para la intervención. Educación XXI, 16(1), 17-38. doi: http://doi.org/10.5944/educXX1.16.1.715

Garaigordobil, M. (2011). Prevalencia y consecuencias del cyberbullying: Una revisión. International Journal of Psychology and Psychosocial Therapy, 11(2), 233-254.

Garaigordobil, M. (2013). Cyberbullying. Screening de acoso entre iguales. Madrid: TEA.

Garaigordobil, M. \& Aliri, J. (2013). Ciberacoso ("Cyberbullying”) en el País Vasco: diferencias de sexo en víctimas, agresores y observadores. Behavioral Psychology/Psicología Conductual, 21(3), 461-474.

Garaigordobil, M. \& Oñederra, J. A. (2008). Bullying: Incidence of peer violence in the schools of the Autonomour Community of the Basque Country. International Journal of Psychology and Psychological Therapy, 8(1), 51-62.

Guarini, A., Brighi, A. \& Genta, M. L. (2009). Traditional Bullying and Cyberbullying in Italian Secondary Schools. En M. L. Genta, A. Brighi \& A. Guarini (Eds.), Bullying and Cyberbullying in Adolescence (pp. 77-95). Roma: Carocci.

Juvonen, J. \& Gross, E. F. (2008). Extending the school grounds? - Bullying experiences in Cyberspace. Journal of School Health, 78 (9), 496-505. doi: https://doi.org/10.1111/j.17461561.2008.00335.x

Li, Q. (2007). Bullying in the New Playground: Research into Cyberbullying and Cyber Victimisation. Australasian Journal of Educational Technology, 23(4), 435-454.

doi: https://doi.org/10.14742/ajet.1245

Livingstone, S., Haddon, L., Görzig, A. \& Ólafsson, K. (2011). Risks and safety on the internet: The perspective of European children. Full Findings. LSE, London: EU
Kids Online.

McKenna, P. (2007). The rise of cyberbullying. The New Scientist, 195 (2613), 26-27. doi: https://doi.org/10.1016/S02624079(07)61835-1

Mora-Merchán, J. A. (2008). Cyberbullying: un nuevo reto para la convivencia en nuestras escuelas. Informació Psicològica, 94, 60-70.

Nocentini, A., Calmaestra, J., SchultzeKrumbholz, A., Scheithauer, H., Ortega, R. \& Menesini, E. (2010). Cyberbullying: Labels, behaviours and definition in three European countries. Australian Journal of Guidance and Counselling, 20(2), 129-142. doi: https://doi.org/10.1375/ajgc.20.2.129

Ortega, R., Calmaestra, J. \& Mora-Merchán, J. A. (2008). Cyberbullying. International Journal of Psychology and Psychological Therapy, 8(2), 183-192.

Pelfrey., W. Jr \& Weber, N. (2013). Keyboard gangsters: analysis of incidence and correlates of cyberbullying in a large urban student population. Deviant Behavior, 34, 6884.

doi: https://doi.org/10.1080/01639625.2012.707541

Perren, S., Dooley, J. J., Shaw, T \& Cross, D. (2010). Bullying in school and cyberspace: Associations with depressive symptoms in Swiss and Australian adolescents. Child and Adolescent Psychiatry and Mental Health, 4(28), 1-10. doi: https://doi.org/10.1186/1753-2000-4-28

Ruiz, R., Riuró, M. \& Tesouro, M. (2015). Estudio de bullying en el ciclo superior de primaria. Educación XXI, 18(1), 345-368. doi:

http://doi.org/10.5944/educXX1.18.1.12384

Sánchez-Lacasa, C. \& Cerezo, F. (2010). Variables personales y sociales relacionadas con la dinámica bullying en escolares de Educación Primaria. Electronic Journal of Research in Educational Psychology, 8(3), 1015-1032.

Smith, P. K., Mahdavi, J., Carvalho, C., Fisher, S., Russell, S. \& Tippett, N. (2008). Cyberbullying: Its nature and impact in secondary school pupils. Journal of Child Psychology and Psychiatry, 49, 376-385. doi: $\quad$ https://doi.org/10.1111/j.1469- 
$\underline{7610.2007 .01846 . x}$

Smith, P. K., Mahdavi, J., Carvalho, M. \& Tippett, N. (2006). An investigation into cyberbullying, its forms, awareness and impact, and the relationship between age and gender in Cyberbullying. (Research Brief No. Brief No: RBX03-06): AntiBullying Alliance.

Viñas, F. (2009). Uso autoinformado de Internet en adolescents: perfil psicológico de un uso elevado de la red. International Journal of Psychology and Psychological Therapy, 9, 109-122.

Wade, A. \& Beran, T. (2011). Cyberbullying: the new era of bullying. Canadian Journal of School Psychology, 26, 44-61. doi: https://doi.org/10.1177/0829573510396318

Willard, N. E. (2006). Cyberbullying and Cyberthreats: Responding to the challenge of online social cruelty, threats and distress.
Eugene, Oregon: Center for Safe and Responsible Internet Use.

Willard, N. E. (2007). The authority and responsibility of school officials in responding to cyberbullying. Journal of Adolescent Health, 41(6, Supplement 1), S64-S65. doi: https://doi.org/10.1016/j.jadohealth.2007.08. $\underline{013}$

Wright, V. H., Burnham, J. J., Inman, C. T. \& Ogorchock, H. N. (2009). Cyberbullying: Using Virtual Scenarios to Educate and Raise Awareness. Journal of Computing in Teacher Education, 26(1), 35-42.

Yilmaz, H. (2011). Cyberbullying in Turkish middle schools: an exploratory study. School Psychology International, 32, 645-654. doi: https://doi.org/10.1177/0143034311410262

\begin{tabular}{|c|c|}
\hline Autores / Authors & $\begin{array}{c}\text { To know more / } \\
\text { Saber más } \\
\end{array}$ \\
\hline Domínguez-Alonso, José (jdalonso@uvigo.es) & ORCID \\
\hline 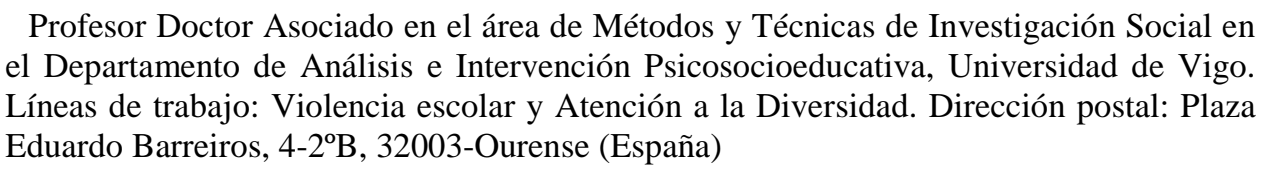 & $\underline{0000-0002-1844-000 X}$ \\
\hline Vázquez-Varela, Elia (eliav@edu.xunta.es) & ORCID \\
\hline $\begin{array}{l}\text { Asesora del Centro de Formación y Recursos. Consellería de Cultura, Educación y } \\
\text { Ordenación Universitaria. Líneas de trabajo: Violencia escolar y Atención a la Diversidad. } \\
\text { Dirección Postal: Plaza de San Antonio, } n^{\circ} 2-7^{\circ}, 32004 \text {, Ourense (España) }\end{array}$ & $\underline{0000-0002-6562-2586}$ \\
\hline \multicolumn{2}{|l|}{ Nuñez-Lois, Sandra (sandranl@edu.xunta.es). } \\
\hline $\begin{array}{l}\text { Directora de Centro de Educación. Consellería de Cultura, Educación y Ordenación } \\
\text { Universitaria. Líneas de trabajo: La voz docente y Atención a la Diversidad. Dirección } \\
\text { Postal: Rúa do Areal, 32002, Ourense (España) }\end{array}$ & \\
\hline
\end{tabular}

\section{RELIEVE}

\section{Revista ELectrónica de Investigación y $\mathbf{E V}$ aluación Educativa E-Journal of Educational Research, Assessment and Evaluation}

[ISSN: 1134-4032]

\footnotetext{
(c) Copyright, RELIEVE. Reproduction and distribution of this articles it is authorized if the content is no modified and their origin is indicated (RELIEVE Journal, volume, number and electronic address of the document).

(C) Copyright, RELIEVE. Se autoriza la reproducción y distribución de este artículo siempre que no se modifique el contenido y se indique su origen (RELIEVE, volumen, número y dirección electrónica del documento).
} 